\title{
Métastases cutanées révélant un cancer du sein aux urgences
}

\section{Cutaneous Metastasis revealing Breast Cancer in the ER}

\author{
F.E. Ekoue Kouvahey $\cdot$ N. Saada $\cdot$ E. Kras
}

Reçu le 18 décembre 2014; accepté le 24 février 2015

(C) SFMU et Lavoisier SAS 2015

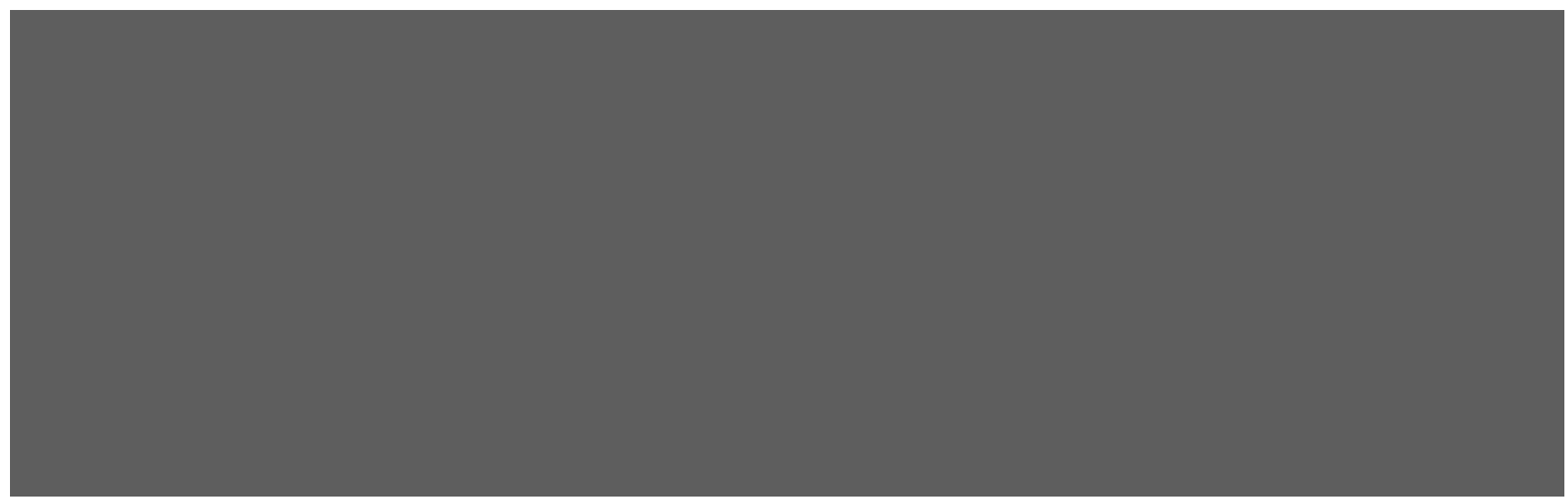

Une femme de 70 ans consulte aux urgences pour une asthénie isolée. L'examen clinique retrouve deux tumeurs cutanées, siégeant sur le cuir chevelu et sur l'abdomen. Elles sont nodulaires, indolores, de consistance dure, dépigmentées, alopéciantes (Fig. 1A) ou hyper-pigmentées, croûteuse, de couleur inhomogène, avec une base inflammatoire (Fig. 1B). Elles ne sont pas fixées aux plans profonds. Le reste des examens cliniques (dont l'examen mammaire) et paracliniques (dont la radiographie pulmonaire) sont sans particularité. Devant la suspicion de malignité, la patiente est orientée dès le service d'accueil des urgences vers un service d'hospitalisation de médecine. Le diagnostic final conclut à des métastases cutanées révélatrices d'un cancer du sein. Les présentations cliniques et les localisations des métastases cutanées sont variées : le caractère multifocal et l'atteinte fréquente du cuir chevelu sont des signes évocateurs. Dans 1 à $2 \%$ des cas, elles sont le signe révélateur d'un cancer viscéral [1]. Les tumeurs primitives les plus souvent incriminées sont le sein et le poumon [2]. La reconnaissance de ces tumeurs cutanées, de diagnostic difficile car nécessitant une inspection minutieuse, permet d'initier, dès les urgences, une prise en charge précoce sur le plan néoplasique.

\section{Références}

1. Azoulay S, Adem C, Pelletier FL, et al (2005) Skin metastases from unknown origin: role of immunohistochemistry in the evaluation of cutaneous metastases of carcinoma of unknown origin. J Cutan Pathol 32: 561-6

2. Riahi R, Cohen P (2012) Clinical manifestations of cutaneous metastases. Am J Clin Dermatol 13:103-12

F.E. Ekoue Kouvahey $\cdot$ N. Saada $\cdot$ E. Kras $(\square)$

Actes Urgences, 6 avenue des peupliers,

F-13960 Sausset-les-Pins

e-mail : etiennekr@hotmail.com 